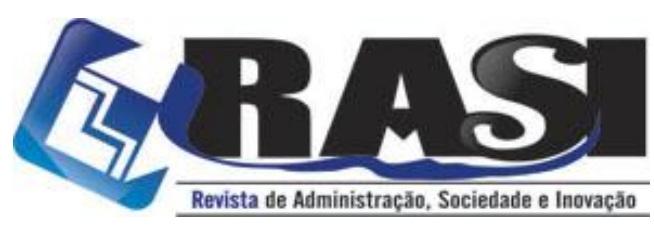

http://www.rasi.uff.br

RASI, Volta Redonda/RJ, v.4, n.1, pp.90-105, jan/jun. 2018

\title{
O Papel da Coopetição na Criação de Valor para Micro e Pequenas Empresas - MPEs no Âmbito da Cadeia de Suprimentos em Relações Fornecedor-Fornecedor
}

Silvio Bitencourt da Silva (UNISINOS) - sibitencourt@unisinos.br Wilciney José Villan (FVA) - neyvillan@hotmail.com

RESUMO: Este ensaio teórico analisa como a coopetição pode criar valor para Micro e Pequenas Empresas MPEs no âmbito da cadeia de suprimentos em relações fornecedor-fornecedor e, por consequência, gerar impactos sobre o desempenho. São conduzidas teorizações pautadas na VBR e uma de suas extensões, a Visão Baseada em Conhecimento - VBC, que indicam que a adoção de estratégias coopetitivas podem acontecer no âmbito da cadeia de suprimentos nas relações fornecedor-fornecedor, que a coopetição tem o papel de promover a busca por recursos que de outra forma seriam inacessíveis e, uma maneira de criar e melhorar a vantagem competitiva. O conhecimento é apresentado como o recurso estratégico mais significativo neste contexto, resultado de diferentes combinações dos tipos de conhecimento (tácito e explícito) e da phronesis. O referencial teórico e as discussões apresentadas suscitam proposições teóricas que servem de ponto de partida para verificações empíricas. Em síntese, apontam para o entendimento de que MPEs são geralmente limitadas em seus recursos e presença no mercado e, que a adoção de estratégias coopetitivas as habilita a obter aprendizado a partir do compartilhamento de conhecimentos existentes ou criação de novos que se transformem em vantagem competitiva, mesmo com seus competidores diretos, criando uma dinâmica fractal.

PALAVRAS-CHAVE: coopetição, conhecimento, recursos, relações fornecedor-fornecedor e micro e pequenas empresas.

\section{The Role of Coopetition in creating value for Micro and small Enterprises - SMEs within the supply chain Sendor-Supplier relationships}

\begin{abstract}
This theoretical essay analyzes how coopetition can create value for Micro and Small Enterprises (SMEs) within the supply chain in supplier-supplier relationships and, consequently, to generate impacts on performance. Resource-based view (RBV) theorizations and one of its extensions, the Knowledge-Based View (KBV), are conducted which indicate that the adoption of coopetition strategies can occur within the supply chain in supplier-supplier relations, which co-participation has the role of promoting the search for resources that would otherwise be inaccessible, and a way to create and improve competitive advantage. Knowledge is presented as the most significant strategic resource in this context, resulting from different combinations of types of knowledge (tacit and explicit) and phronesis. The theoretical framework and the discussions presented raise theoretical propositions that serve as a starting point for empirical verifications. In summary, they point to the understanding that MPEs are generally limited in their resources and presence in the market and that the adoption of coopetition strategies enables them to obtain learning from the sharing of existing knowledge or creation of new ones that become competitive advantage, even with its direct competitors, creating a dynamic fractal.
\end{abstract}

KEYWORDS: coopetition, knowledge, resources, supplier-supplier relationships and micro and small companies.

\section{Universidade
Federal
Fluminense}

R. Desembargador Ellis Hermydio Figueira, 783, Bloco A, sl. 304, Aterrado.

27213-145 - Volta Redonda, RJ - Brasil www.uff.br

Copyright (C) 2018 RASI. Todos os direitos, até mesmo de tradução, são reservados. É permitido citar parte de artigos sem autorização prévia, desde que seja identificada a fonte 


\section{O Papel da Coopetição na Criação de Valor para Micro e Pequenas Empresas - MPEs no Âmbito da Cadeia de Suprimentos em Relações Fornecedor-Fornecedor}

\section{Introdução}

A pesquisa sobre coopetição em que se combinam definições de competição e de cooperação (Dagnino \& Padula, 2002) em um ambiente altamente competitivo (Kotzab \& Teller, 2003) tem obtido crescente atenção em pesquisa (Ketchen, Snow \& Hoover, 2004). Seu conceito é derivado do trabalho de Brandenburger e Nalebuff (1996) que discorriam sobre a teoria dos jogos e suas relações com as estratégias de organizações.

A coopetição tem por finalidade trazer benefícios para os envolvidos e são consideradas como uma maneira eficiente de lidar simultaneamente com a cooperação e a competição entre competidores (Bengtsson \& Kock, 2000) que compartilhem características semelhantes e sejam, portanto, similares (Das \& Teng, 2000), além de terem visão e objetivos comuns (Zineldin, 2004).

De acordo como Bouncken et al. (2015) quando as empresas pretendem aumentar mutuamente os lucros da indústria e criar um mercado maior para seus produtos, optam pela coopetição como estratégia para a criação de valor. Logo que o valor é criado as mesmas empresas se voltam umas contra as outras para apropriar o máximo valor possível por conta própria (Bouncken, Gast, Kraus \& Bogers, 2015). A obtenção deste valor maior (Rusko, 2011) ou, ao menos, uma situação vantajosa para todas as partes através de um mercado maior (Liu, 2013) são motivos principais que levam empresas concorrentes a adotar estratégias coopetitivas.

Dois dos contextos específicos em que a estratégias coopetitivas acontecem são de particular interesse para a construção teórica deste estudo, permitindo a delimitação do debate que segue: o das Micro e Pequenas Empresas - MPEs (Levy, Loebbecke \& Powell, 2003; Morris, Koçak \& Özer, 2007; Robert, Marques \& Le Roy, 2009; Thomason, Simendinger \& Kiernan, 2013) e o da gestão da cadeia de suprimentos (Gurnani, Erkoc \& Luo, 2007; Fulconis, Hiesse \& Paché, 2011; Li, Liu \& Liu, 2011; Sepehri \& Fayazbakhsh, 2011).

O contexto das MPEs parece ser uma promissora, mas subexplorada possibilidade de pesquisa (Bouncken et al., 2015), pois apenas um número limitado de estudos tem focado explicitamente nas potenciais contribuições da coopetição no contexto das MPEs, tais como Levy et al. (2003), Morris et al. (2007) e Thomason et al. (2013).

Devido ao seu tamanho, as MPEs são geralmente limitadas em seus recursos e presença no mercado (Morris et al., 2007). Assim, ao adotarem estratégias coopetitivas podem melhorar sua base de recursos, sua posição competitiva nos mercados existentes e sua entrada em mercados novos ou estrangeiros (Bouncken et al., 2015).

A adoção de estratégias coopetitivas pode acontecer no âmbito da cadeia de suprimentos nas relações comprador-fornecedor e fornecedor-fornecedor como se observa em estudos apontados por Bouncken et al. (2015), tais como Gurnani et al. (2007), Eriksson (2008) e Lacoste (2012). Neles, segundo Bouncken et al. (2015), se exploram diferentes questões, como, por exemplo, o papel da coopetição e a criação de conhecimento na cadeia de suprimentos como no trabalho de Wilhelm (2011).

Nesta situação, foi determinada a seguinte questão norteadora para o debate neste ensaio teórico: qual o papel da coopetição na criação de valor para MPEs no âmbito da cadeia de suprimentos em relações fornecedor-fornecedor? 
Neste sentido, este estudo pretende lançar as bases para futuros estudos empíricos, particularmente voltados a compreensão das estratégias coopetitivas de MPEs e do conhecimento criado em torno do fornecimento no atacado para lojistas, como por exemplo através de centros atacadistas.

Uma das perspectiva teóricas que possibilita o delineamento da compreensão das interações coopetitivas é a Visão Baseada em Recursos - VBR (Garcia \& Velasco, 2002; M'Chirgui, 2005) já apontada como uma oportunidade de pesquisa por Walley (2007). A oferta de recursos únicos assegura a atratividade de um ator para que outros atores se associem a este para o desenvolvimento de um objetivo comum (Garcia\& Velasco, 2002; M'Chirgui, 2005).

A partir da VBR, a cooperação com concorrentes se torna uma busca por recursos que de outra forma seriam inacessíveis e uma maneira de criar e melhorar a vantagem competitiva, como é possível observar em estudos que exploram esta perspectiva (Morris et al., 2007; Bonel \& Rocco, 2009; Choi, Garcia \& Friedrich, 2010; Gnyawali \& Park, 2009; 2011)

Esta reflexão está alinhada as atuais tendências e novas perspectivas de pesquisa e contextos teóricos e empíricos sobre coopetição, como observado por Gast, Filser, Gundolf, \& Kraus (2015), Bouncken et al. (2015) e Bengtsson, Raza-Ullah e Vanyushyn (2016).

Para alcançar o objetivo proposto, este trabalho compreendido como um ensaio teórico, qualitativo, exploratório e bibliográfico (Martins \& Theóphilo, 2007) busca criar condições explicativas da realidade à luz do referencial adotado, suscitando discussões por meio da identificação de lacunas na literatura, da integração de teorias e da identificação de novas possibilidades ou contextos para sua interpretação.

O artigo apresenta, além desta introdução, as teorizações sobre coopetição, VBR, e uma de suas extensões, a Visão Baseada em Conhecimento VBC, além dos tipos de conhecimento para sustentação do debate acerca da criação de conhecimento no contexto das MPEs nas relações fornecedor-fornecedor. Na sequência, são apresentadas proposições que oferecem ao campo direções de pesquisa, algumas considerações finais e, por fim, as referências utilizadas.

\section{Método}

Para a elaboração deste ensaio teórico foi conduzido primeiramente o levantamento bibliográfico de caráter exploratório, selecionando através da ferramenta Web of Science, artigos que continham as palavras-chave "coopetition" e "resource-based view", "coopetition" e "knowledge-based view", "coopetition + resource" e "coopetition + knowledge" no tópico. A pesquisa preliminar destacou 178 artigos internacionais na área de administração.

Como nenhum artigo nacional foi localizado, se realizou nova pesquisa no Portal de Periódicos CAPES. Na busca por assunto "coopetição" e "visão baseada em recursos", "coopetição" e "visão baseada em conhecimento", "coopetiação + recursos" e "coopetição + conhecimento", tendo sido identificados onze artigos, sendo que somente dois deles eram pertinentes.

$\mathrm{Na}$ sequencia, se fez a síntese e elaboração do referencial teórico, onde se desenvolve uma integração de três teorias, a visão baseada em recursos, a visão baseada em conhecimento e a gestão estratégica do conhecimento, integradas dentro de uma relação de coopetição em uma cadeia de suprimento, na interação fornecedor/fornecedor. 


\section{Referencial Teórico}

\subsection{Coopetição}

No âmbito acadêmico a coopetição é explorada inicialmente por Brandenburger e Nalebuff (1996) como uma nova forma de pensar os negócios por meio de relações multifacetadas entre empresas concorrentes, fornecedores, complementadores e clientes.

A coopetição é um jogo de múltiplos ganhadores que competem e cooperam para obter benefícios mútuos, o qual é considerado um comportamento coerente dentro de um sistema econômico (Padula \& Dagnino, 2007) e, de modo evolutivo, um sistema ou uma estratégia (Dagnino \& Rocco, 2009; Dagnino, Di Guardo \& Padula, 2012) que pode se tornar em uma fonte de vantagem competitiva para as empresas (Barney, 2011; Barney, Della Corte, Sciarelli \& Arikan, 2012).

Uma das definições usualmente adotadas na literatura é dada por Zineldin (2004, p.780) que a apresenta como a "situação de negócio em que independentes partes cooperam entre si e coordenam as suas atividades, dessa forma colaborando para alcançar objetivos mútuos, mas ao mesmo tempo, competindo entre si, bem como com outras empresas".

No entanto, pesquisas sobre coopetição têm sido conduzidas há mais de duas décadas e vários conceitos requerem uma apreciação mais precisa, pois a literatura mostra que há uma falta de definições unificadas, uma vez que várias definições foram empregadas na pesquisa realizada anteriormente (Bengtsson \& Kock, 2014).

Para os autores Bengtsson e Kock (2014) a definição precoce de coopetição, como uma relação dupla entre empresas que simultaneamente cooperam e competem, precisaria ser refinada. Sugerem que a coopetição é "uma relação paradoxal entre dois ou mais atores, independentemente de estarem em relações horizontais ou verticais, envolvidas simultaneamente em interações cooperativas e competitivas" (Bengtsson \& Kock, 2014, p. 180).

Com base no amplo conjunto de dimensões da coopetição sugerido na literatura, Bouncken et al. (2015) propõem a seguinte definição integrativa: "a coopetição é um processo estratégico e dinâmico no qual os atores econômicos criam valor conjuntamente através da interação cooperativa, ao mesmo tempo que competem para captar parte desse valor".

Assim, a coopetição representa interconexões mais complexas frente às relações tradicionais competitivas e cooperativas autônomas, especialmente quando associadas ao desenvolvimento e desdobramento do conhecimento (Levy et al, 2003; Luo, Slotegraaf \& Pan, 2006) e, portanto, requerem reflexões mais detalhadas (Bengtsson \& Kock, 2014; Della Corte \& Aria, 2014; 2016).

Em uma revisão conduzida por Bengtsson, Raza-Ullah e Vanyushyn (2016) que tenta sintetizar a pesquisa sobre coopetição durante um período de tempo de 18 anos a coopetição é definida de forma diferente e ocorre em diferentes níveis. Duas escolas de pensamentos são identificadas por meio do desenvolvimento de um modelo multinível abrangente e dinâmico que as integra: a escola do ator que enfoca o contexto da rede de coopetição e a escola de atividades de pensamentos voltada as relações de coopetição entre o mesmo par de empresas.

Eles argumentam que os drivers e os resultados são os mesmos dentro de ambas as escolas, mas os processos de coopetição em redes e em relações diádicas diferem. Eles sugerem uma perspectiva mista sobre a coopetição que nos permite entender como as relações de coopetição e o contexto da rede se afetam mutuamente e o próprio resultado da coopetição. 
Para Bouncken et al. (2015) a coopetição é mais do que uma simples palavra "buzz" ou apenas outra forma de pesquisa de alianças, descrevendo as crescentes relações cooperativas interorganizacionais entre empresas concorrentes. $\mathrm{O}$ autor afirma que se trata de um fluxo aceito e crescente de pesquisa por conta própria na intersecção da estratégia e de vários campos de pesquisa. No entanto, é necessária investigação adicional para explorar a sua conceitualização e sua aplicabilidade estratégica, bem como a sua gestão em diferentes contextos (Bouncken et al., 2015).

A complexidade das interconexões no âmbito da coopetição exige troca, desenvolvimento e desdobramento de capacidades e recursos aptos à configurar o contexto de coopetição, bem como o desenvolvimento de vantagem competitiva para os envolvidos. Deste modo, a identificação da disponibilidade, ou não, destes recursos e capacidades que serão envolvidos no contexto de coopetição, envolvem ações gerenciais que ditam a forma de compartilhamento e proteção dos recursos, dado que sua classificação é distinta entre as empresas.

\subsection{Visão Baseada em Recursos - VBR}

A VBR é uma lente teórica que se desenvolveu a partir das perspectivas econômicas de Penrose (1959) e se refere a um conjunto de proposições que, a partir de uma lógica econômica, estabelece um modelo para reflexão sobre a disponibilidade, ou não, de recursos e capacidades que possibilitem a obtenção de vantagem competitiva.

Através da visão baseada em recursos (RBV), o acesso a recursos e capacidades únicas, valiosas, inimitáveis e não substituíveis (Barney, 1991; Grant, 1991) pode ser uma fonte de vantagem competitiva.

Nesse contexto, de acordo com Barney (1991) e Barney, Wright e Ketchen (2001) os recursos são os ativos tangíveis e intangíveis que uma empresa utiliza para escolher e programar suas estratégias e, segundo Amit e Schoemaker (1993) podem ser compreendidos como recursos e capacidades.

Duas suposições básicas estão implícitas na abordagem baseada em recursos: (1) que os recursos e capacidades podem variar significativamente entre as empresas (a suposição da heterogeneidade dos recursos) (2) de uma maneira estável (a suposição de imobilidade dos recursos) (Barney, 1991; Peteraf, 1993).

Além disso, quatro atributos que definem o potencial de recursos da empresa para gerar vantagem competitiva sustentável são propostos por Barney (1991), sendo eles: valioso (Barney, 1986a; 1991), raro (Barney, 1986a;1991), inimitável (Barney, 1986b; 1991; Peteraf, 1993) e insubstituível (Dierickx \& Cool, 1989; Barney, 1991).

Para que um recurso da empresa tenha o potencial de gerar vantagem competitiva sustentável, deve, simultaneamente, exibir cada um dos quatro atributos propostos (Barney, 1991), pois mesmo que individualmente necessários tais atributos sozinhos não são suficientes (Dierickx \& Cool, 1989; Priem \& Butler, 2001).

No contexto empresarial, a partir do entendimento amplamente apoiado empiricamente (Crook, Ketchen, Combs \& Todd, 2008) de que na VBR os recursos da empresa têm influência sobre o seu desempenho, nota-se que não há resultado apenas pela posse de recursos, mas também é necessária a ação gerencial sobre o portfólio de recursos da empresa (Helfat et al., 2007; Sirmon, Hitt \& Ireland, 2007).

Dessa forma, uma das críticas à VBR está voltada à forma com que são desenvolvidos recursos e capacidades para a obtenção de vantagem competitiva, pois estes não surgiriam naturalmente (Kraaijenbrink, Spender \& Groen, 2010). Ao contrário, o seu desenvolvimento 
deve ser conduzido ativamente pelos gestores (Helfat et al., 2007; Mahoney, 1995; Sirmon et al., 2007).

Em termos evolutivos, como um campo de estudo, a VBR tem obtido destaque e alcançado relevância no campo da estratégia, ao se constituir na mais importante contribuição para a disciplina, especialmente a partir da década de 90 quando assegurou amplo reconhecimento entre os pesquisadores da área (Ramos-Rodríguez \& Ruíz-Navarro, 2004).

Atualmente, a VBR parece ter atingido a maturidade como uma teoria, encontrando-se em um momento crítico, que seria seguido por sua revitalização ou por seu declínio (Barney, Ketchen \& Wright, 2011). Demonstra, ainda, perspectivas de crescimentos (Lu \& Liu, 2013) especialmente nos temas que se propõem a expandir a VBR como os sugeridos por Barney, Wright e Ketchen (2001): interligações com outras perspectivas, processos de aquisição e desenvolvimento de recursos, as microfundações da VBR, a relação entre VBR e a sustentabilidade, e questões de método e mensuração.

Uma das possibilidades de expansão se refere a Visão Baseada no Conhecimento VBC que suscita novos discernimentos a partir do entendimento de que o conhecimento é o recurso mais significativo da empresa, e apresentados na subseção seguinte.

\subsubsection{Visão Baseada no Conhecimento -VBC}

Na VBC é sugerido que a razão primária da empresa é a criação e aplicação do conhecimento (Demsetz, 1991; Grant, 1996a; Spender, 1996). Nesta direção, diferentes desempenhos entre empresas são resultado de diferentes bases de seus conhecimentos e diferentes capacidades no desenvolvimento e desdobramento do conhecimento (Bierly \& Chakrabarty,1996). Para Eisenhardt e Santos (2002) e Grant (1996a) a VBC considera o conhecimento como o recurso mais significativo da empresa.

As capacidades da firma para lidar com o conhecimento são as principais abordagens para desenvolver vantagem competitiva baseada em conhecimento. As formas de transmitir conhecimento, absorver, torná-lo em algo de valor ou que agregue valor ao negócio, e, por fim, a coordenação destas capacidades formam a base para desenvolver esta vantagem competitiva (Grant, 1996a).

Distinções entre os conhecimentos tácitos e explícitos são feitas na literatura, porém a VBC considera o conhecimento tácito como o mais importante devido ao seu armazenamento em indivíduos altamente especializados. Deste modo o papel principal da organização é integrar este conhecimento, por meio, principalmente, da rotina e direcionamento, tornando-o uma capacidade organizacional, assim, esta capacidade é estruturada hierarquicamente consoante ao propósito do conhecimento (Grant, 1996b).

A vantagem competitiva baseada no conhecimento impacta as estratégias de inovação das organizações. Inovações em processos são originadas internamente à organização, são mais caras para implementar, porém, mais eficazes em relação à inovação de produtos e, fortalecem a vantagem competitiva originada por intermédio do uso do conhecimento nos processos de inovação (Gopalakrishnan, Bierly \& Kessler, 1999).

A utilização deste recurso possibilita a geração de inovações, bem como a criação de um ciclo virtuoso de inovações e melhorias, e ampara as escolhas da organização quanto ao posicionamento no ambiente competitivo (Fialho, Santos, Macedo \& Mitidieri, 2006).

Neste sentido, o conhecimento como um bem intangível, impacta o desempenho organizacional (Nonaka \& Takeuchi, 1997). A gestão do conhecimento deve integrar a estratégia da organização, dado que o resultado é a agregação de valor às informações e origem de melhores práticas de administração dos recursos organizacionais. É por intermédio 
desta prática que se agrega mais valor aos bens intangíveis da organização (Ponchirolli \& Fialho, 2005).

Compreendendo o conhecimento como o recurso mais significativo da empresa, a cooperação entre firmas ganha espaço em âmbito competitivo para a obtenção de aprendizado a partir do compartilhamento de conhecimentos existentes ou criação de novos conhecimentos (Tsai, 2002; Luo et al., 2006) que se transformem em vantagem competitiva, mesmo com seus competidores diretos (M'Chirgui, 2005; Teece \& Pisano, 1994).

Nesta direção, Loebbecke e Angehrn (2003) apontam para os componentes fundamentais de aprendizagem coopetitiva e redes de troca de conhecimento. Neste âmbito, o conhecimento advém, além dos dados e informações, dos processos de aprendizagem colaborativa; dado que o trabalhador e a organização devem ser interpretados como agentes dotados de capacidades de possuir e processar conhecimento; a troca de conhecimento entre os agentes acontecem entre as redes pessoais, mas também entre organizações; o aumento das redes interorganizacionais estimula a atenção sobre os processos de aprendizagem e troca de conhecimento entre os atores envolvidos no ambiente de coopetição; as trocas de conhecimento entre organizações se baseia na cooperação; o conhecimento é visto como um recurso utilizado pra obtenção de vantagem competitiva; e as trocas de conhecimento permitem a criação de valor.

Todavia, a gestão e compartilhamento do conhecimento num ambiente de coopetição é complexo. Assim, inovação nas configurações da gestão do conhecimento neste âmbito, geram benefícios aos envolvidos (Loebbecke \& Angehrn, 2011).

A literatura que aborda a gestão do conhecimento aponta para a distinção dos tipos de conhecimento. Conhecer os tipos de conhecimento, bem como o contexto em que ele é criado, se desenvolve e é compartilhado possibilita seu gerenciamento e possibilita o desenvolvimento de vantagem competitiva. Deste modo os tipos de conhecimento e suas combinações são abordados a seguir.

\subsection{Tipos de conhecimento}

A literatura trata de dois tipos de conhecimento e suas combinações. O conhecimento explícito, aquele que pode ser transmitido formalmente; e o conhecimento tácito, visto como altamente pessoal e de difícil formalização (Polanyi \& Grene, 1969).

A vantagem competitiva conquistada por meio deste recurso, originário dos indivíduos, ocorre por intermédio de sua criação e disseminação em toda a organização, além de sua rápida incorporação em tecnologias, produtos, processos etc. Neste âmbito, os conhecimentos tático e explícito se relacionam em forma de espiral, dado que a criação do conhecimento ocorre por meio de combinações entre estes tipos, ou seja, por meio de articulação e internalização (Nonaka, 1991; 1994).

Todavia, a criação deste conhecimento ocorre em algum lugar. Nonaka e Konno (1998) utilizam o conceito de "Ba" para caracterizar este lugar. O termo pode ser entendido como um local, um contexto ou um espaço compartilhado em movimento, seja ele físico, virtual, mental ou uma combinação entre eles, no qual é próprio para a criação do conhecimento individual ou coletivo.

A combinação dos tipos de conhecimento (tácito e explícito) possibilita a identificação de padrões distintos de formação de conhecimento: tácito-tácito; tácito-explícito; explícitoexplícito; explícito-tácito. Essas combinações, dada pela espiral do conhecimento, ocorre em um "Ba", o qual é derivado do tipo de combinação feito entre os dois tipos de conhecimento. 
Em uma companhia, há diferentes organizações, cada uma delas, detém características relacionadas à um dos padrões descritos, assim o "Ba" é construído pelos esforços da organização. A priorização de uma relação dos tipos de conhecimento, caracterizam um "Ba".

Visando o alcance de vantagem competitiva por meio de uma companhia inovativa, Nonaka, Kodama, Hirose \& Kohlbacher (2013) apresentam um novo conceito de organização, chamada de "Organização Fractal" baseada em uma dinâmica formação de "Ba", para alcançar uma síntese ente a criação de um novo conhecimento e a exploração de um conhecimento existente. A integração em espiral dos dois tipos de conhecimento criam a terceira parte do conhecimento: a phronesis. Assim, a dinâmica de síntese de criação de um novo conhecimento e a exploração de um conhecimento existente é realizada através da dialética entre conhecimento tácito, explicito e a sabedoria prática (phronesis).

Deste modo, para os autores, a dinâmica da organização fractal ocorre em um ambiente (ecossistema do conhecimento) no qual uma organização mantém a troca constante de conhecimento tácito e explicito, criando a phronesis. Esta troca ocorre, ainda, dentro de um contexto em movimento ("Ba"). Neste mesmo ecossistema há outras organizações realizando o mesmo processo. A proximidade dos contextos (dos "Bas") sintetiza o conhecimento das duas organizações. Assim, esta síntese é um fractal das duas organizações, o qual representa o todo. Este processo, a relação entre os conhecimentos, é dividido através das organizações, e é esta dinâmica que constrói a organização fractal.

Neste contexto, a integração do conhecimento é variável entre as organizações, assim, diferentes combinações de conhecimento impactam as possibilidades de integração do conhecimento entre diferentes organizações, empresas ou unidades de negócios. Pari passu à interligação do conhecimento e da aprendizagem, diferentes formas, métodos e ações de criação e compartilhamento de conhecimento são necessários para gerar aprendizagem correlata (Andreu \& Sieber, 2005).

Em âmbito organizacional, a cadeia de suprimentos das empresas contempla o conhecimento obtido por intermédio de seus membros, o qual sua gestão é um conjunto de competências constituído por aquisição, conversão, aplicação e proteção do conhecimento. Assim, os conhecimentos tácito e explícito proporcionam a manifestação da capacidade de gerenciamento do conhecimento na cadeia de suprimentos, vista como uma rotina interorganizacional e uma capacidade dinâmica capaz de produzir resultados favoráveis (Schoenherr, Griffith \& Chandra, 2014).

No contexto empresarial a estratégia se configura de diferentes formas. A coopetição, como uma destas configurações, estimula a competição e a cooperação simultaneamente para o desenvolvimento de vantagem competitiva. Integra-se neste âmbito relações internas e externas à organização. Entre elas, o conhecimento como recurso estratégico. A seguir, discute-se a importância da coopetição para a melhoria de uma base de recursos, na qual o conhecimento é abordado como recurso mais significativo para as organizações.

\section{Teorização e Proposiçôes}

A seguir, a Figura 1 representa o esquema sugerido em relação a como a coopetição pode criar valor para MPESs no âmbito da cadeia de suprimentos em relações fornecedorfornecedor. Sugere-se que o papel da coopetição na criação de valor para MPEs no âmbito da cadeia de suprimentos em relações fornecedor-fornecedor se refere a melhoria de sua base de recursos que de outra forma seriam inacessíveis, sendo o conhecimento o recurso estratégico 
mais significativo neste contexto, resultado de diferentes combinações dos tipos de conhecimento (tácito e explícito) e da phronesis.

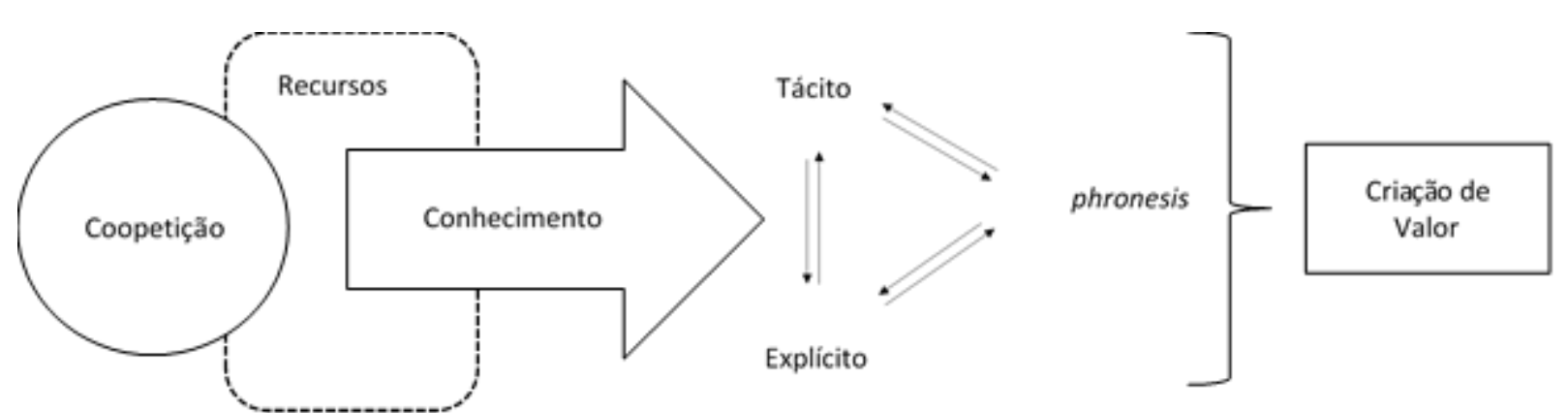

Figura 1. Papel da coopetição na criação de valor Fonte: Elaborado pelos autores (2017).

Dessa forma, o referencial teórico e as discussões apresentadas suscitam algumas proposições teóricas que servem de ponto de partida para verificações empíricas sobre qual o papel da coopetição na criação de valor para MPEs no âmbito da cadeia de suprimentos em relações fornecedor-fornecedor. São quatro as proposições:

Proposição 1: Os recursos, caracterizados como valiosos, raros, inimitáveis e insubstituíveis, como caracterizados por Barney, (1986a; b; 1991), Peteraf, (1993) e Dierickx e Cool, (1989) são protegidos pelas companhias com a finalidade de alcançar vantagem competitiva sustentável. Todavia em um ambiente de coopetição recursos são compartilhados, dado que neste ambiente há muitas empresas que competem e cooperam para obter benefícios mútuos (Padula \& Dagnino, 2007). Assim, a adoção de estratégias coopetitivas pelas MPEs no âmbito da cadeia de suprimentos nas relações fornecedor-fornecedor, podem melhorar sua base de recursos, que de outra forma seriam inacessíveis, além de criar e melhorar a vantagem competitiva.

Proposição 2: Consequentemente, o conhecimento como recurso mais significativo da empresa, também é compartilhado, exigindo das companhias capacidades para transmitir, absorver e usar conhecimento para agregar valor ao negócio. Deste modo, coordenar estas capacidades possibilitam o desenvolvimento de vantagem competitiva (Grant, 1996a). À vista disso, a adoção de estratégias coopetitivas pelas MPEs no âmbito da cadeia de suprimentos nas relações fornecedor-fornecedor permite a criação e aplicação de conhecimento em um processo de aprendizagem coopetitiva, onde todos aprendem além de cooperarem e competirem simultaneamente.

Proposição 3: Contemplando o conhecimento como recurso que afeta a vantagem competitiva dos atores envolvidos por intermédio da coopetição, o contexto coopetitivo também sofre alterações por consequência da transmissão, absorção e uso 
do conhecimento, ou seja, o ambiente é moldado constantemente à medida que o conhecimento é compartilhado. Consequentemente as relações de coopetição e o contexto da rede de MPEs no âmbito da cadeia de suprimentos nas relações fornecedor-fornecedor se afetam mutuamente além do resultado da coopetição.

Proposição 4: A troca de conhecimento entre os envolvidos no âmbito da coopetição, pressupõe que cada um deles estão inseridos em seus respectivos contextos dinâmicos (Nonaka e Konno, 1998). Nestes contextos a relação constante dos tipos de conhecimento (tácito, explícito e da phronesis) criam, entre as diferentes organizações, uma dinâmica fractal (Nonaka et al., 2013), ou seja, criam uma dinâmica formação de locais, contextos ou um espaços compartilhados, para alcançar uma síntese ente a criação de um novo conhecimento e a exploração de um conhecimento existente. Deste modo, ao atuarem simultaneamente numa estratégia de coopetição, os contextos dinâmicos de diferentes companhias/empresas se encontram, aumentando as relações de conhecimento tácito, explícito e phronesis. Logo, o encontro destes contextos advindos de companhias diferentes no âmbito da cadeia de suprimentos nas relações fornecedor-fornecedor caracterizam uma nova dinâmica fractal entre as organizações e companhias.

A partir das proposições apresentadas, sustenta-se que o compartilhado de conhecimento por meio da estratégia de coopetição influencia a criação e melhoria da vantagem competitiva, a criação de valor, o resultado da coopetição e caracteriza uma nova dinâmica fractal entre os envolvidos. A partir destas proposições a sessão seguinte considera, de forma mais ampla, as abordagens teóricas em relação à cadeia de suprimentos nos vínculos fornecedor-fornecedor.

\section{Considerações Finais e Direções de Pesquisa}

Com base nas discussões apresentadas neste ensaio teórico, se analisou como a coopetição pode criar valor para MPESs no âmbito da cadeia de suprimentos em relações fornecedor-fornecedor e, por consequência, gerar impactos sobre o desempenho. Para este fim, se discutiu o exercício da coopetição como uma alternativa a adoção das tradicionais estratégias de competição e cooperação a partir da perspectiva teórica reconhecida como VBR.

Foram conduzidas teorizações pautadas na VBR e uma de suas extensões, a VBC, que indicaram que a adoção de estratégias coopetitivas pode acontecer no âmbito da cadeia de suprimentos nas relações fornecedor-fornecedor e que a coopetição tem o papel de promover a busca por recursos que de outra forma seriam inacessíveis e uma maneira de criar e melhorar a vantagem competitiva.

O conhecimento foi apresentado como o recurso estratégico mais significativo neste contexto, resultado de diferentes combinações dos tipos de conhecimento (tácito e explícito) e da phronesis. A priorização de uma relação dos tipos de conhecimento, expressa o valor criado.

O referencial teórico e as discussões apresentadas suscitaram algumas proposições teóricas que servem de ponto de partida para verificações empíricas. Em síntese, se obteve o entendimento de que MPEs são geralmente limitadas em seus recursos e presença no mercado, e ao adotarem estratégias coopetitivas, se habilitam a obter aprendizado a partir do 
compartilhamento de conhecimentos existentes ou criação de novos que se transformem em vantagem competitiva, mesmo com seus competidores diretos, criando uma dinâmica fractal.

Por meio das lacunas teóricas, este trabalho apresentou como resultados proposições teóricas que servem de base para testes empíricos. Com isso busca-se avançar na discussão referente às teorias abordadas neste trabalho, visto que diferentes formas de obter vantagem competitiva são abordadas na literatura. Deste modo ainda são incipientes discussões que relacionam coopetição, visão baseada em recursos e principalmente visão baseada em conhecimento.

Em síntese, esta abordagem apresenta a possibilidade de novos estudos aplicados dentro da cadeia de suprimentos, na aplicação do modelo proposto neste ensaio teórico. Neste sentido, o próprio modelo pode, posteriormente, ser implementado e revisado por meio de sua aplicação prática.

Como sugestão para futuras investigações neste campo de estudo, sugere-se a realização de estudos empíricos sobre a adoção de estratégias coopetitivas no âmbito da cadeia de suprimentos em relações fornecedor-fornecedor de MPES voltadas a validação ou refutação das proposições apresentadas.

\section{REFERÊNCIAS}

Amit, R., \& Schoemaker, P. J. (1993). Strategic assets and organizational rent. Strategic management journal, 14(1), 33-46.

Andreu, R., \& Sieber, S. (2005). Knowledge integration across organizations: how different types of knowledge suggest different 'integration trajectories'. Knowledge and Process Management, 12(3), 153-160.

Barney, J. B., Della Corte, V., Sciarelli, M., \& Arikan, A. (2012). The role of resource-based theory in strategic management studies: Managerial implications and hints for research. Handbook of research on competitive strategy, 109-146.

Barney, J. B. (1991). Firm resources and sustained competitive advantage. Journal of management, 17(1), 99-120.

Barney, J. B. (2011). Gaining and sustaining competitive advantage (4th Edition). Upper Saddle River, NJ: Prentice Hall.

Barney, J. B. (1986a). Strategic factor markets: Expectations, luck, and business strategy. Management science, 32(10), 1231-1241.

Barney, J. B. (1986b). Organizational culture: can it be a source of sustained competitive advantage?. Academy of management review, 11(3), 656-665.

Barney, J. B., Ketchen Jr, D. J., \& Wright, M. (2011). The future of resource-based theory: revitalization or decline?. Journal of management, 37(5), 1299-1315.

Barney, J. B., Wright, M., \& Ketchen Jr, D. J. (2001). The resource-based view of the firm: Ten years after 1991. Journal of management, 27(6), 625-641.

Bengtsson, M., \& Kock, S. (2000). " Coopetition" in business Networks - to cooperate and compete simultaneously. Industrial marketing management, 29(5), 411-426. 
Bengtsson, M., \& Kock, S. (2014). Coopetition-Quo vadis? Past accomplishments and future challenges. Industrial Marketing Management, 43(2), 180-188.

Bengtsson, M., Raza-Ullah, T., \& Vanyushyn, V. (2016). The coopetition paradox and tension: The moderating role of coopetition capability. Industrial Marketing Management, 53, 19-30.

Bierly, P., \& Chakrabarti, A. (1996). Generic knowledge strategies in the US pharmaceutical industry. Strategic management journal, 17(S2), 123-135.

Bonel, E., \& Rocco, E. (2009). Coopetition and business model change-A case-based framework of coopetition-driven effects. Coopetition strategy theory, experiments and cases, 191-218.

Bouncken, R. B., Gast, J., Kraus, S., \& Bogers, M. (2015). Coopetition: a systematic review, synthesis, and future research directions. Review of Managerial Science, 9(3), 577-601.

Brandenburger, A. M., \& Nalebuff, B. J. Co-opetition. New York: Doubleday., 1996.

Choi, P., Garcia, R., \& Friedrich, C. (2010). The drivers for collective horizontal coopetition: a case study of screwcap initiatives in the international wine industry. International Journal of Strategic Business Alliances, 1(3), 271-290.

Crook, T. R., Ketchen, D. J., Combs, J. G., \& Todd, S. Y. (2008). Strategic resources and performance: a meta-analysis. Strategic management journal, 29(11), 1141-1154.

Dagnino, G. B.; Di Guardo, M. C., \& Padula, G. (2012). Coopetition: Nature, challenges, and implications for firms' strategic behavior and managerial mindset. In G. B. Dagnino (ed.). Handbook of research on competitive strategy (pp.492-511). Northamptom, MA: Edward Elgar.

Dagnino, G. B., \& Padula, G. (2002, May). Coopetition strategy: a new kind of interfirm dynamics for value creation. In Innovative research in management, European Academy of Management (EURAM), second annual conference, Stockholm, May (Vol. 9).

Dagnino, G. B., \& Rocco, E. (2009). Coopetition strategy. London: Routledge.

Das, T. K., \& Teng, B. S. (2000). Instabilities of strategic alliances: An internal tensions perspective. Organization science, 11(1), 77-101.

Della Corte, V., \& Aria, M. (2016). Coopetition and sustainable competitive advantage. The case of tourist destinations. Tourism Management, 54, 524-540.

Della Corte, V., \& Aria, M. (2014). Why strategic networks often fail: Some empirical evidence from the area of Naples. Tourism Management, 45, 3-15.

Demsetz, H. (1991). The Theory of the Firm Revisited. In: O. E. Williamson \& S. G. Winter (eds.), The Nature of the Firm. New York (pp.159-178). Oxford University Press.

Dierickx, I., \& Cool, K. (1989). Asset stock accumulation and sustainability of competitive advantage. Management science, 35(12), 1504-1511. 
Eisenhardt, K., \& Santos F. (2002). Knowledge-Based View: A New Theory of Strategy? In: A. Pettigrew, H. Thomas, \& R. Whittington, (eds.), Handbook of Strategy and Management. London: Sage Publications.

Eriksson, P. E. (2008). Achieving suitable coopetition in Buyer-Supplier relationships: The case of AstraZeneca. Journal of Business-to-Business Marketing, 15(4), 425-454.

Fialho, F. A. P., Santos, N., Macedo, M., \& Mitidieri, T. (2006). Gestão do conhecimento e aprendizagem: as estratégias competitivas da sociedade pós-industrial. Florianópolis: Visual Books.

Fulconis, F., Hiesse, V., \& Paché, G. (2011). The 3PL Provider as Catalyst of Coopetitive Strategies: An Exploratory Study. In Supply Chain Forum: An International Journal, 12(2), p. 58-69.

Garcia, C. Q., \& Velasco, C. A. (2002, May). Co-opetition and performance: evidence from European biotechnology industry. In II Annual Conference of EURAM on 'Innovate Research in Management', Stockholm (Sweden).

Gast, J., Filser, M., Gundolf, K., \& Kraus, S. (2015). Coopetition research: towards a better understanding of past trends and future directions. International Journal of Entrepreneurship and Small Business, 24(4), 492-521.

Gnyawali, D. R., \& Park, B. J. R. (2009). Co-opetition and technological innovation in small and medium-sized enterprises: A multilevel conceptual model. Journal of small business management, 47(3), 308-330.

Gnyawali, D. R., \& Park, B. J. R. (2011). Co-opetition between giants: Collaboration with competitors for technological innovation. Research Policy, 40(5), 650-663.

Gopalakrishnan, S., Bierly, P., \& Kessler, E. H. (1999). A reexamination of product and process innovations using a knowledge-based view. The Journal of High Technology Management Research, 10(1), 147-166.

Grant, R. M. (1991). The resource-based theory of competitive advantage: implications for strategy formulation. California management review, 33(3), 114-135.

Grant, R. M. (1996a). Toward a knowledge-based theory of the firm. Strategic management journal, 17, 109-122.

Grant, R. M. (1996b). Prospering in dynamic competitive environments: organizational capability as knowledge integration. Organizational Science, 7(4), 375-387.

Gurnani, H., Erkoc, M., \& Luo, Y. (2007). Impact of product pricing and timing of investment decisions on supply chain co-opetition. European Journal of Operational Research, 180(1), 228-248.

Helfat, C., Finkelstein, S., Mitchell, W., Peteraf, M., Singh, H., Teece, D., \& Winter, S. (2007). Dynamic capabilities: understanding strategic change in organizations. Malden, MA: Blackwell.

Ketchen, D. J., Jr., Snow, C. C., \& Hoover, V. L. (2004). Research on competitive dynamics: recent accomplishments and future challenges. Journal of Management, 30(6), 779-804. 
Kotzab, H., \& Teller, C. (2003). Value-adding partnerships and co-opetition models in the grocery industry. International Journal of Physical Distribution \& Logistics Management, 33(3), 268-281.

Kraaijenbrink, J., Spender, J. C., \& Groen, A. J. (2010). The resource-based view: A review and assessment of its critiques. Journal of management, 36(1), 349-372.

Lacoste, S. (2012). "Vertical coopetition": The key account perspective. Industrial Marketing Management, 41(4), 649-658.

Levy, M., Loebbecke, C., \& Powell, P. (2003). SMEs, co-opetition and knowledge sharing: the role of information systems. European Journal of Information Systems, 12(1), 3-17.

Li, Y., Liu, Y., \& Liu, H. (2011). Co-opetition, distributor's entrepreneurial orientation and manufacturer's knowledge acquisition: Evidence from China. Journal of Operations Management, 29(1), 128-142.

Liu, R. (2013). Cooperation, competition and coopetition in innovation communities. Prometheus, 31(2), 91-105.

Loebbecke, C., \& Angehrn, A. (2003). Investigating coopetitive learning and knowledge exchange Networks (CoLKENs) as emerging concept in management literature and practice. In Proceedings of the Fourth Conference on Organizational Knowledge, Learning and Capabilities.

Loebbecke, C., \& Angehrn, A. (2011). Knowledge management under coopetition. In Organizational learning and knowledge: Concepts, methodologies, tools and applications (pp. 1192-1205). IGI Global.

Lu, L. Y., \& Liu, J. S. (2013). An innovative approach to identify the knowledge diffusion path: the case of resource-based theory. Scientometrics, 94(1), 225-246.

Luo, X., Slotegraaf, R. J., \& Pan, X. (2006). Cross-functional "coopetition": The simultaneous role of cooperation and competition within firms. Journal of Marketing, 70(2), 67-80.

M'Chirgui, Z. (2005). The economics of the smart card industry: towards coopetitive strategies. Economics of Innovation and New Technology, 14(6), 455-477.

Mahoney, J. T. (1995). The management of resources and the resource of management. Journal of business research, 33(2), 91-101.

Martins, G. A., \& Theóphilo, C. R. (2007). Metodologia da Investigação Científica para Ciências Sociais Aplicadas. São Paulo: Atlas.

Morris, M. H., Koçak, A., \& Özer, A. (2007). Coopetition as a small business strategy: Implications for performance. Journal of small business strategy, 18(1), 35-55.

Nonaka, I. (1994). A dynamic theory of organizational knowledge creation. Organization science, $5(1), 14-37$.

Nonaka, I., Kodama, M., Hirose, A., \& Kohlbacher, F. (2013). Dynamic fractal organizations for promoting knowledge-based transformation-A new paradigm for organizational theory. European Management Journal, 32(1), 137-146. 
Nonaka, I., \& Konno, N. (1998). The concept of" ba": Building a foundation for knowledge creation. California management review, 40(3), 40-54.

Nonaka, I., \& Takeuchi, H. (1997). Criação de conhecimento na empresa: como as empresas japonesas geram a dinâmica da inovação. 20. ed. Rio de Janeiro: Elsevier.

Nonaka; I. (1991). The knowledge-creating company. Harvard Business Review, NovemberDecember, p.96-104.

Padula, G., \& Dagnino, G. B. (2007). Untangling the rise of coopetition: the intrusion of competition in a cooperative game structure. International Studies of Management \& Organization, 37(2), 32-52.

Penrose, E. (1959). The theory of the growth of the firm. Oxford: Basil Blackwell.

Peteraf, M. A. (1993). The cornerstones of competitive advantage: A resource-based view. Strategic management journal, 14(3), 179-191.

Polanyi, M., \& Grene, M. G. (1969). Knowing and Being Essays.

Ponchirolli, O., \& Fialho, F. A. P. (2016). Gestão estratégica do conhecimento como parte da estratégia empresarial. Revista da FAE, 8(1).

Priem, R. L., \& Butler, J. E. (2001). Is the resource-based "view" a useful perspective for strategic management research?. Academy of management review, 26(1), 22-40.

Ramos-Rodríguez, A. R., \& Ruíz-Navarro, J. (2004). Changes in the intellectual structure of strategic management research: A bibliometric study of the Strategic Management Journal, 1980-2000. Strategic Management Journal, 25(10), 981-1004.

Robert, F., Marques, P., \& Le Roy, F. (2009). Coopetition between SMEs: an empirical study of French professional football. International Journal of Entrepreneurship and Small Business, 8(1), 23-43.

Rusko, R. (2011). Exploring the concept of coopetition: a typology for the strategic moves of the Finnish forest industry. Industrial Marketing Management, 40(2):311-320.

Schoenherr, T., Griffith, D. A., \& Chandra, A. (2014). Knowledge management in supply chains: The role of explicit and tacit knowledge. Journal of Business Logistics, 35(2), 121135 .

Sepehri, M., \& Fayazbakhsh, K. (2011). A quantitative examination of competition, coopetition and cooperation in supply chains. South African Journal of Business Management, 42(3), 61-70.

Sirmon, D. G., Hitt, M. A., \& Ireland, R. D. (2007). Managing firm resources in dynamic environments to create value: Looking inside the black box. Academy of management review, 32(1), 273-292.

Spender, J. C. (1996). Making knowledge the basis of a dynamic theory of the firm. Strategic management journal, 17, 45-62.

Teece, D., \& Pisano, G. (1994). The dynamic capabilities of firms: an introduction. Industrial and corporate change, 3(3), 537-556. 
Thomason, S. J., Simendinger, E., \& Kiernan, D. (2013). Several determinants of successful coopetition in small business. Journal of Small Business \& Entrepreneurship, 26(1), 15-28.

Tsai, W. (2002). Social structure of "coopetition" within a multiunit organization: Coordination, competition, and intraorganizational knowledge sharing. Organization science, 13(2), 179-190.

Walley, K. (2007). Coopetition: an introduction to the subject and an agenda for research. International Studies of Management \& Organization, 37(2), 11-31.

Wilhelm, M. M. (2011). Managing coopetition through horizontal supply chain relations: Linking dyadic and network levels of analysis. Journal of Operations Management, 29(7), 663-676.

Zineldin, M. (2004). Co-opetition: the organisation of the future. Marketing Intelligence \& Planning, 22(7), 780-790. 